\title{
Projective Synchronization of Bidirectionally Coupled Chaotic Systems via Linear Transformations
}

\author{
Mohammad Ali Khan ${ }^{1}$, Swarup Poria ${ }^{2}$ \\ ${ }^{1}$ Department of Mathematics, Garhbeta Ramsundar Vidyabhaban, \\ West Bengal, India \\ E-mail: mdmaths@gmail.com \\ ${ }^{2}$ Department of Applied Mathematics, University of Calcutta, \\ Kolkata, West Bengal, India \\ E.mail: swarup_p@yahoo.com
}

\begin{abstract}
Several important properties of chaos synchronization of bidirectionally coupled systems remain still unexplored. This paper designes a new projective synchronization scheme for generalized bidirectionally coupled chaotic systems via linear transformations. The proposed synchronization scheme of bidirectionally coupled chaotic systems are discussed taking coupled unified chaotic systems. Simulation results are presented to show the effectiveness of the proposed scheme.
\end{abstract}

Keywords: Projective synchronization(PS), Unified chaotic system.

\section{Introduction}

Since the pioneer work by Pecora and Caroll [1], chaos synchronization has received much attention because of its fundamental importance in non-linear dynamics and potential applications to laser dynamics, electronic circuits, chemical and biological systems and secure communication. Many chaos synchronization and control methods have been developed, such as backstepping design method [2], impulsive control method [3], invariant manifold method [4], adaptive control method [5], active control method [6] etc. Synchronization in unidirectionally coupled systems and bidirectionally coupled systems are reported by many authors [7-9]. 
Given a pair of dynamical systems

$$
\begin{aligned}
& \dot{X}=f(X) \\
& \dot{Y}=g(Y)
\end{aligned}
$$

are said to be unidirectionally coupled if

$$
\begin{aligned}
\dot{X} & =f(X) \\
\dot{Y} & =g(Y)+h(X, Y)
\end{aligned}
$$

where $h(X, Y)$ is a nontrivial function of $X$ and $Y$.

Physically, this means that in part of the phase space, the behavior of one system has no influence on the behavior of the other. If coupling is not unidirectional then it must be bidirectional. Systems are bidirectionally coupled if

$$
\begin{aligned}
& \dot{X}=f(X)+k(X, Y) \\
& \dot{Y}=g(Y)+h(X, Y)
\end{aligned}
$$

where $h(X, Y)$ and $k(X, Y)$ are nontrivial functions of $X$ and $Y$.

Two dynamical systems are called synchronized if the distance between the corresponding states of the systems converges to zero as time goes to infinity. This type of synchronization is known as identical synchronization [1]. However in the coupled chaotic systems identical synchronization only occurs at a certain point in the system parameter space, and thus is a fairly restrictive concept and often difficult to achieve except under ideal conditions. Recently, a more elaborate form of synchronization called projective synchronization (PS) was proposed by Mainieri and Rahacek in 1999 [10].They observed that projective synchronization in coupled chaotic systems where the drive and response vectors synchronize upto a constant ratio $\alpha$ (scaling factor). Identical synchronization and anti-phase synchronization are the special cases of projective synchronization where $\alpha=1$ and $\alpha=-1$ respectively. Controlled projective synchronization in non-partiallylinear chaotic systems was investigated by Li Z et.al. [11]. In 2004 Li et.al.[12] have reported a necessary condition of projective synchronization in discrete-time systems of arbitrary dimensions. Generalized projective synchronization of a unified chaotic system was proposed by Li. et.al.[13] in 2005. In 2006 GuoHui[14] proposed generalized projective synchronization of two chaotic systems by using active control. Generalized projective synchronization of unidirectionally coupled chaotic resource systems was investigated by Chen et.al.[15] and Cai. et.al.[16]. There are still very few results about synchronization of bidirectionally coupled chaotic systems. But most of the natural systems are bidirectionally 
coupled. Therefore the study of bidirectionally coupled systems are necessary. Nobody have discussed the PS of two bidirectionally coupled chaotic systems via linear transformation. In this paper we introduce the theory of PS of two bidirectionally coupled chaotic systems via linear transformations. Sufficient conditions for PS of two bidirectionally coupled chaotic systems are derived. We discuss the theory considering two bidirectionally coupled Unified chaotic systems. Finally simulation results are presented and discussed.

\section{Projective Synchronization of Bidirectionally Coupled Systems}

A dynamical system can be decomposed into two parts

$$
\dot{X}=A X+\Psi(X)
$$

where $\mathrm{A}$ is an $n \times n$ constant matrix and $\Psi: R^{n} \rightarrow R^{n}$ function. We assume that the driving system transmit the signal $\Psi(X)$ to the driven system and consider the following bidirectional scheme:

$$
\begin{array}{ll}
\dot{X}=A X+\Psi(X)+\Phi(Y) & \text { driving system } \\
\dot{Y}=A Y+\Lambda \Psi(X)+\Lambda \Phi(Y) & \text { driven system }
\end{array}
$$

where A is constant matrix and $\Lambda$ is multiple of identity matrix and $\Psi, \Phi: R^{n} \rightarrow R^{n}$ function.

Theorem 1: If the matrix $\Lambda$ commutes with $A$, then the two coupled chaotic dynamical systems in (5) are in a state of projective synchronization via the following linear transformation

$$
Y(\infty)=H(X)=\Lambda X
$$

if and only if all eigenvalues of the matrix $A$ have negative real parts.

Proof: Let $Z=Y-\Lambda X$, then the stability of the PS between the two dynamical system in (5) via the PS transformation $Y=H(X)=\Lambda X$ is equivalent to that of the origin of the following system:

$$
\begin{aligned}
& \dot{Z}=[A Y+\Lambda \Psi(X)+\Lambda \Phi(Y)]-[\Lambda A X+\Lambda \Psi(X)+\Lambda \Phi(Y)] \\
& =A Y-\Lambda A X \\
& =A(Y-\Lambda X) \quad \text { since } \Lambda \text { commutes with } A \\
& =A Z .
\end{aligned}
$$


Therefore $Z=0$ is asymptotically stable if and only if all eigen values of the matrix $A$ have negative real parts.

\section{Projective Synchronization of Bidirectionally Coupled Unified Chaotic Systems}

In this section we discuss the theory considering two bidirectionally coupled Unified chaotic systems via linear transformations. A unified chaotic system is presented by $\mathrm{Lu}$ and $\mathrm{Wu}$ in 2004 [17]. The Unified chaotic system can be described by the following system of differential equations.

$$
\begin{aligned}
& \dot{\mathrm{x}}=(25 \mathrm{a}+10)(\mathrm{y}-\mathrm{x}) \\
& \dot{\mathrm{y}}=(28-35 \mathrm{a}) \mathrm{x}-\mathrm{xz}+(29 \mathrm{a}-1) \mathrm{y} \\
& \dot{\mathrm{z}}=\mathrm{xy}-\frac{8+\mathrm{a}}{3} \mathrm{z}
\end{aligned}
$$

where $a \in[0,1]$. For $a=0,0.8,1$ the system (7) represents the Lorenz chaotic system, Lu chaotic system and Chen chaotic system respectively. Practically, Unified chaotic system is chaotic for any $a \in[0,1]$. According to equation (5) the driving and driven systems with bidirectional coupling are constructed as

Driving system :

$$
\begin{aligned}
& \dot{\mathrm{x}}_{1}=(25 \mathrm{a}+10)\left(\mathrm{x}_{2}+\mathrm{y}_{2}-\mathrm{x}_{1}\right) \\
& \dot{\mathrm{x}}_{2}=(28-35 \mathrm{a}) \mathrm{x}_{1}-\mathrm{x}_{2}-\mathrm{x}_{1} \mathrm{x}_{3}-\mathrm{y}_{1} \mathrm{y}_{3}+29 \mathrm{a}\left(\mathrm{x}_{2}+\mathrm{y}_{2}\right) \\
& \dot{\mathrm{x}}_{3}=\mathrm{x}_{1} \mathrm{x}_{2}+\mathrm{y}_{1} \mathrm{y}_{2}-\frac{8+\mathrm{a}}{3} \mathrm{x}_{3}
\end{aligned}
$$

Driven system:

where

$$
\begin{gathered}
\dot{y}_{1}=-(25 a+10) y_{1}+\lambda(25 a+10)\left(x_{2}+y_{2}\right) \\
\dot{y}_{2}=(28-35 a) y_{1}-y_{2}+\lambda\left\{29 a\left(x_{2}+y_{2}\right)-x_{1} x_{3}-y_{1} y_{3}\right\} \\
\dot{y}_{3}=-\frac{8+a}{3} y_{3}+\lambda\left(x_{1} x_{2}+y_{1} y_{2}\right) \\
\Lambda=\left[\begin{array}{lll}
\lambda & 0 & 0 \\
0 & \lambda & 0 \\
0 & 0 & \lambda
\end{array}\right]
\end{gathered}
$$




\section{Simulation Results}

Numerical simulations have been performed for demonstration of the synchronization method. Fourth-order Runge-Kutta method is used for solving bidirectionally coupled Unified chaotic system with step size .001. The initial conditions for the driving and driven system are taken as $\left(x_{1}(0), x_{2}(0), x_{3}(0)\right)=(.02, .02, .02)$ and $\left(y_{1}(0), y_{2}(0), y_{3}(0)\right)=(.02, .02, .02)$.

We take

$$
\Lambda=\left(\begin{array}{lll}
\lambda & 0 & 0 \\
0 & \lambda & 0 \\
0 & 0 & \lambda
\end{array}\right) \text { and } \mathrm{A}=\left[\begin{array}{ccc}
-(25 a+10) & 0 & 0 \\
(28-35 a) & -1 & 0 \\
0 & 0 & -\frac{8+a}{3}
\end{array}\right]
$$

where $\lambda \neq 0$. Clearly $\Lambda A=A \Lambda$. Therefore all conditions of the theorem are satisfied. Here the driving Unified chaotic system is (8) and the driven Unified chaotic system is given by

$$
\begin{aligned}
\dot{\mathrm{y}}_{1} & =(25 \mathrm{a}+10)\left(\lambda\left(\mathrm{x}_{2}+\mathrm{y}_{2}\right)-\mathrm{y}_{1}\right) \\
\dot{y}_{2} & =(28-35 a) y_{1}-y_{2}+\lambda\left(-x_{1} x_{3}-y_{1} y_{3}+29 a\left(x_{2}+y_{2}\right)\right) \\
\dot{y}_{3} & =\lambda\left(x_{1} x_{2}+y_{1} y_{2}\right)-\frac{8+a}{3} y_{3}
\end{aligned}
$$

The simulation results of synchronization are shown in Figures 1(a-c) and errors are shown in Figure1(d). We choose $\lambda=2$, then the state variables of the driving system and driven system are connected by the linear transformation

$$
\begin{aligned}
& y_{1}=2 x_{1} \\
& y_{2}=2 x_{2} \\
& y_{3}=2 x_{3}
\end{aligned}
$$

We choose $\lambda=-1$, then the state variables of the driving system and driven system are connected by the linear transformation

$$
\begin{aligned}
& y_{1}=-x_{1} \\
& y_{2}=-x_{2} \\
& y_{3}=-x_{3}
\end{aligned}
$$

The results of anti-phase synchronization are shown in Figures 2(a-c) and the errors are shown in Figure 2(d).

We choose $\lambda=1$, then the state variables of the driving system and driven system are connected by the linear transformation

$$
\begin{aligned}
& y_{1}=x_{1} \\
& y_{2}=x_{2} \\
& y_{3}=x_{3}
\end{aligned}
$$


The results of identical-synchronization are shown in Figure 3(a-c) and the errors are shown in Figure 3(d).
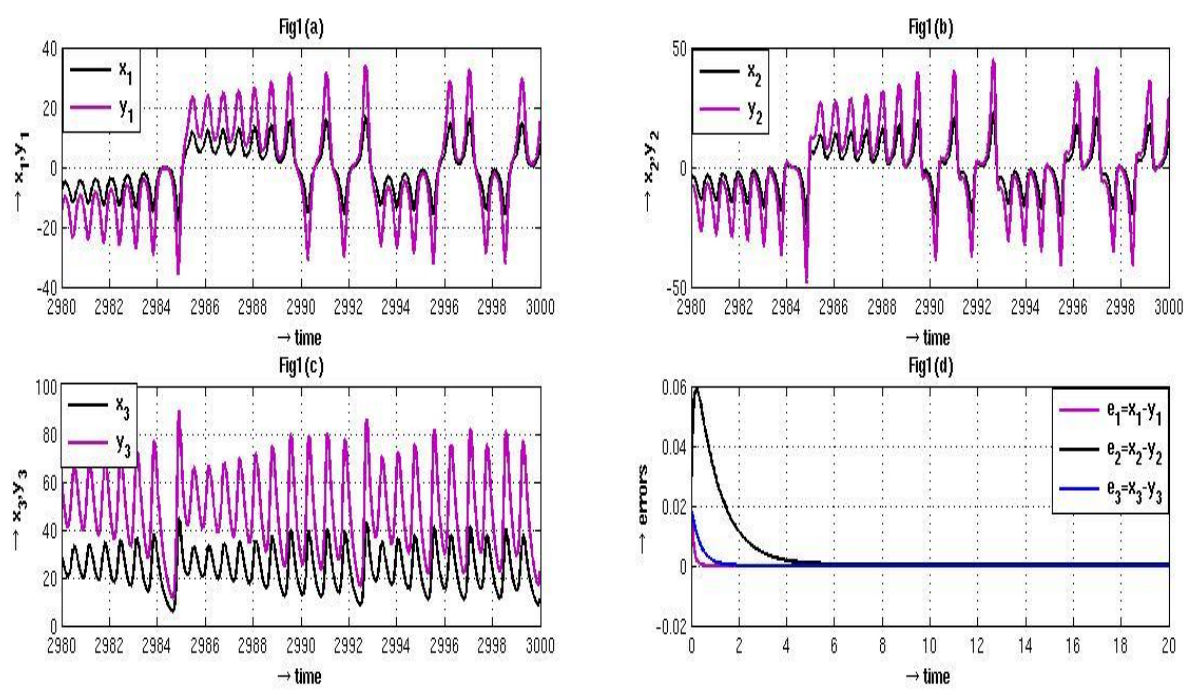

Fig1(a-d). Time evolution of $\mathrm{x}_{1}$ and $\mathrm{y}_{1}$ states (Fig1(a)), $\mathrm{x}_{2}$ and $\mathrm{y}_{2}$ states (Fig1(b)), $\mathrm{x}_{3}$ and $\mathrm{y}_{3}$ states (Fig1(c)) and errors (Fig1(d)) for bidirectionally coupled unified chaotic systems for $\mathrm{a}=0$ i.e., in the Lorenz systems.
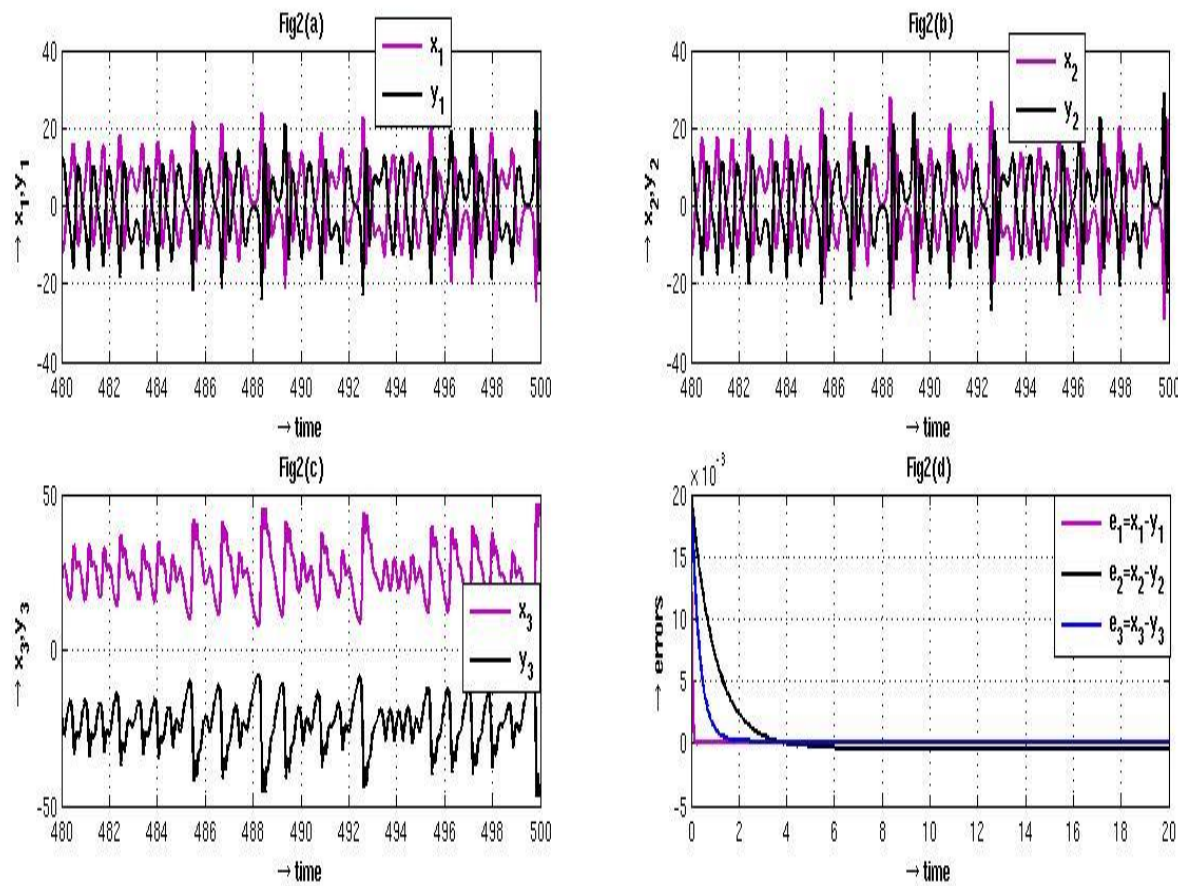

Fig2(a-d). Time evolution of $\mathrm{x}_{1}$ and $\mathrm{y}_{1}$ states (Fig2(a)), $\mathrm{x}_{2}$ and $\mathrm{y}_{2}$ states (Fig2(b)), $\mathrm{x}_{3}$ and $\mathrm{y}_{3}$ states (Fig2(c)) and errors (Fig2(d)) for bidirectionally coupled unified chaotic systems for $\mathrm{a}=0.8$ i.e., in the Lu systems. 

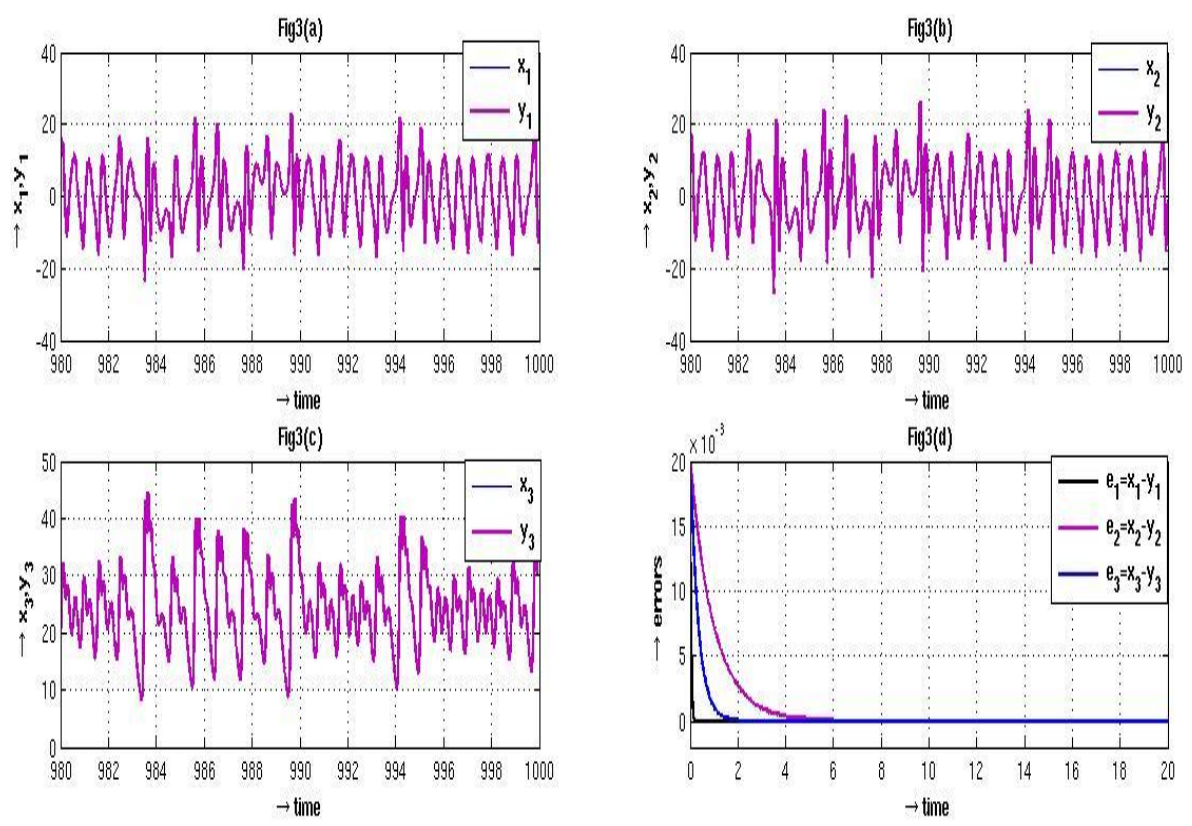

Fig3(a-d). Time evolution of $\mathrm{x}_{1}$ and $\mathrm{y}_{1}$ states (Fig3(a)), $\mathrm{x}_{2}$ and $\mathrm{y}_{2}$ states (Fig3(b)), $\mathrm{x}_{3}$ and $\mathrm{y}_{3}$ states (Fig3(c)) and errors (Fig3(d)) for bidirectionally coupled unified chaotic systems for $\mathrm{a}=1$ i.e., in the Chen systems.

Firstly we obtain projective synchronization of coupled Lorenz systems $(a=0)$. Secondly anti-phase synchronization of coupled Lu systems $(\mathrm{a}=0.8)$ and lastly identical synchronization of coupled Chen systems $(\mathrm{a}=1)$ are presented

\section{Conclusions}

We have proposed a scheme for projective synchronization of bidirectionally coupled chaotic systems via linear transformation. We discuss the theory considering coupled Unified chaotic systems. Our numerical simulation results shows the efficiency of the proposed scheme. We have obtained identical synchronization and anti-phase synchronization for special type of coupling. This scheme may be useful for sending secrete message and to understand synchronization of many biological systems and it can be used to extend binary digital to M-nary digital communication for achieving fast communication.

\section{References}

[1] L.M. Pecora and T.L. Carroll, Synchronization in chaotic systems, Phys. Rev. Lett., 64(1990) 821-824. 
[2] Y. Zhang, Controlling uncertain Lu system using backstepping design, Chaos, Solitons and Fractals., 15(2003) 897-902.

[3] T. Yang, L-B. Yang, C-M. Yang, Impulsive control of Lorenz system, Physica D., 110(1997)18-24.

[4] Y. Song, Chaos synchronization via controlling partial state of chaotic systems, Int. J. Bifurcat. Chaos., 1(2001)1737.

[5] Chen Lu, Synchronization of an uncertain unified chaotic system via adaptive control, Chaos, Solitons and Fractals., 23(2005) 1319-1325.

[6] MC Ho and YC Hung, Synchronization of two different systems by using generalized active control, Physics Letters A., 301(2002) 424-428.

[7] M.A. Khan and A.K.Mandal, Generalized chaos synchronization of coupled Rossler systems, Bull. Cal. Math. Soc., 101(2009)197-204.

[8] A. Tarai(Poria), S. Poria and P. Chatterjee, Synchronization of bidirectionally coupled chaotic Chen's system with delay, Chaos, Solitons and Fractals., 41(2009) 643-647.

[9] A. Tarai(Poria), S. Poria and P. Chatterjee, Synchronization of generalized linearly bidirectionally coupled unified system, Chaos, Solitons and Fractals., 40(2009) 885-892.

[10] M. Ronnie and R. Jan , Projective synchronization in three-dimensional chaotic systems, Phys. Rev. Lett., 82(1999) 3042-3045.

[11] $\mathrm{Xu} \mathrm{D}$ and Li Z, Controlled projective synchronization in nonpartially-linear chaotic systems, Int. J. Bifurcat. Chaos.,12(2002) 1395-1402.

[12] $\mathrm{Xu} \mathrm{D}$, Chee $\mathrm{C}$ and $\mathrm{Li} \mathrm{C}$, A necessary condition of projective synchronization in discrete-time systems of arbitrary dimensions, Chaos Solitons and Fractals.,22(2004) 175-180.

[13] J. Yan and C. Li, Generalized projective synchronization of a unified chaotic system, Chaos Solitons and Fractals, 26(2005) 1119-1124.

[14] Guo-Hui Li, Generalized projective synchronization of two chaotic systems using active control, Chaos Solitons and Fractals., 30(2006)77-82.

[15] Hua Chen and Mei Sun, Generalized projective synchronization of the energy resource system, Int. J. of Nonlinear science., 2(2006)166-170.

[16] G Cai and S Zheng, Projective synchronization of a new chaotic system.Journal of uncertain systems., 2(2007) 67-74.

[17] $\mathrm{Lu}$ and $\mathrm{Wu}, \mathrm{A}$ unified chaotic system with continuous periodic switch, Chaos, Solitons and Fractals., 20(2004) 245-251. 